\title{
GCU
}

Glasgow Caledonian

University

University for the Common Good

\section{A computational intelligence based dislocation recognition during molecular dynamics simulation}

Huang, Jianfeng; Chen, Yi ; Luo, Yuaxin; McGlinchey, Don

Published in:

22nd International Conference on Automation and Computing (ICAC)

DOI:

10.1109/IConAC.2016.7604923

Publication date:

2016

Document Version

Author accepted manuscript

Link to publication in ResearchOnline

Citation for published version (Harvard):

Huang, J, Chen, Y, Luo, Y \& McGlinchey, D 2016, A computational intelligence based dislocation recognition during molecular dynamics simulation. in 22nd International Conference on Automation and Computing (ICAC). IEEE, 22nd International Conference on Automation and Computing (ICAC), Essex, United Kingdom, 7/09/16. https://doi.org/10.1109/IConAC.2016.7604923

\section{General rights}

Copyright and moral rights for the publications made accessible in the public portal are retained by the authors and/or other copyright owners and it is a condition of accessing publications that users recognise and abide by the legal requirements associated with these rights.

Take down policy

If you believe that this document breaches copyright please view our takedown policy at https://edshare.gcu.ac.uk/id/eprint/5179 for details of how to contact us. 


\section{A Computational Intelligence based Dislocation Recognition During Molecular Dynamics Simulation}

\author{
Yuanxin Luo \\ State Key Lab of \\ Mechanical Transmission \\ Chongqing University, Chongqing, \\ 400044, China \\ Email:yxluo@cqu.edu.cn
}

\author{
Jianfeng Huang \\ and Don McGlinchey \\ School of Engineering and \\ Built Environment \\ Glasgow Caledonian University \\ Glasgow, U.K. G4 0BA \\ Email: jianfeng.huang@gcu.ac.uk \\ Email: D.McGlinchey@gcu.ac.uk
}

\author{
Yi Chen* \\ College of Computer Science \\ and Technology \\ Dongguan University of Technology \\ Dongguan 523808,China \\ Email: chenyi@dgut.edu.cn
}

\begin{abstract}
Dislocation evolution in metal is the majority response of external stress or strain during deformation. In this paper, a computational intelligence aided dislocation recognition algorithm which is integrated in a molecular dynamics simulation is proposed. This algorithm is inspired by genetic algorithm(GA) and follows the main GA process in which the initial population is the disperse nodes set and is under the select, crossover to evolve the new generation of nodes set. Finally, an example of the dislocation recognition during fatigue simulation with this algorithm is provided with good result by comparing with the traditional MD post process.
\end{abstract}

\section{INTRODUCTION}

Fatigue crack initiation which associated with the dislocation evolution in crystalline material causes the life of material decrease sharply. Thus, understanding of the dislocation and its evolution is essential in the material fracture from the atomic scale point of view. Although, with the different types of fatigue, high cycle fatigue(HCF) and low cycle fatigue(LCF), the magnitude and frequency of external loading is different. For this reason, the material response of the different external loading is different. But the fatigue crack initiation begins with the same process at the atomic level - the broken of the interatomic bond. In theory, the critical stress of material in this situation is much greater than that of the experiment. The expression is $\sigma_{c} \approx \frac{E}{\pi}$ where $E$ is Young's modulus. Take iron for example, where $E=210 G P a$, the theoretical critical stress is about $70 G P a$ which could only valid for defect-free single crystal iron. Thus, in the real situation of polycrystalline material with defects, whose critical stress is $2-3$ orders of magnitude less than the theoretical one, it's important to understand the effect of defects on the material fatigue and fracture, especially, dislocation.

According to the importance of the dislocation in crystalline structure deformation, the knowledge of the dislocation is essential for revealing the nature of material fatigue and fracture. The term of dislocation was coined by Taylor [1] in 1934. After that, Researchers focused on the relation between the plasticity of crystalline material with the dislocation. And experiment examination of the material revealed the dislocation exist commonly during material deformation. Those measurements include scanning electron microscope(SEM), transmission electron microscopy(TEM) and field ion microscope(FIM). The research on the dislocation and other material defects by the observation technologies rich people's knowledge enhanced theorem of material fracture in the viewpoint of material science.

In molecular dynamics simulation, the lattice structure depends on the topology between atoms. For example, the the bond between atoms in face-centred cubic(FCC) lattice, which is a typical Bravais lattice, is shown in Fig. 1. And the atom bond in the potential area is defined as Equation 1 in which $F$ is a force function of the distance between atom $i$ and its nearby atoms in a predefined cutoff range. To detect the atom defect, many methods has been published and this is reviewed in section III. The theory proof of those methods is nearly based on the bond between neighbour atoms or the distance between neighbour atoms.

$$
\Phi_{i}=\sum_{i=0}^{n} F\left(r_{i}\right)
$$

In this paper, based on the traditional MD post analysis method, a computational intelligence aided dislocation recognition method has been stated. In section II, the genetic algorithms, which are a part of computational intelligence, are briefly introduced. Following this, the traditional MD post analysis methods which used to analysis crystal defects such as dislocation and stack fault is reviewed in detail. Later, in section IV, the GA-based dislocation recognition algorithm is presented with detail workflow and then the result is provided in section $\mathrm{V}$. 


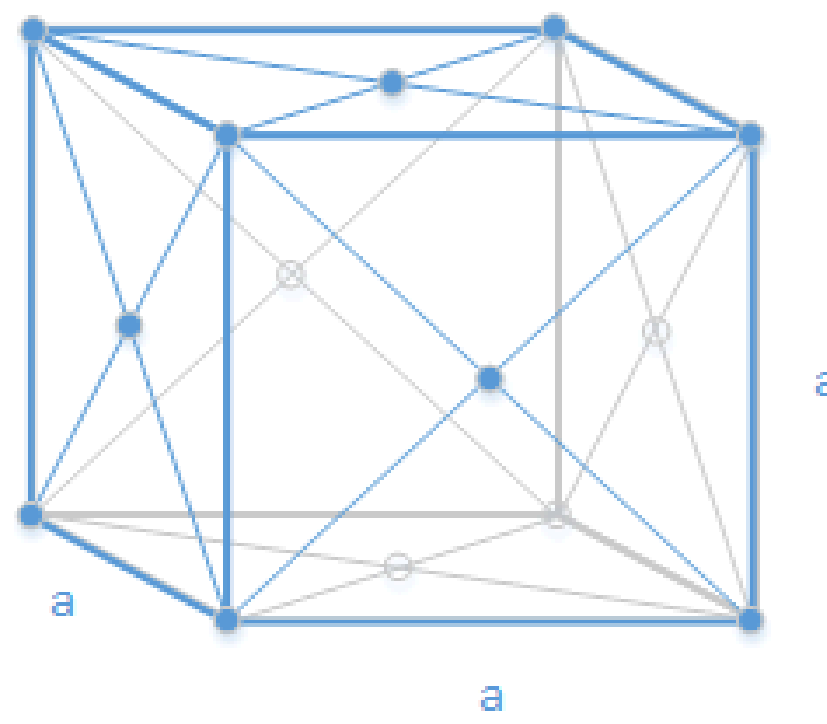

Fig. 1. FCC Lattice

\section{Computational Intelligence Application}

Although Darwin's evolutionary theory is a crisis now in biological science, but this evolution theory and the genetics theory which funded by Mendel inspired the Genetic Algorithms (GAs) in artificial intelligence. The forerunner of GAs is John Holland [2] in the 1970s and then popularised by him and his students [3]. The performance of GAs in complex domain search had been proven to be efficiency and powerful so that the reorganisation of the problem which is searching a nonlinear and non-differentiable space is not needed. And also, multiple local optima target can be set during GAs searching while some other optimization algorithms are single target [4]. What's more, the fitness score of GAs, obtained from objective functions, can be expressed as a proper function of the system's output, for example, a polynomial function. The important is that the GAs are parallel computing algorithms which have better performance than others. Using the GAs, the engineering optimal criteria can be represented by the fitness functions [5].

As depicted in Figure 2, the initial population should be coded for each individual at first. After the selection of population with the fitness function, some replicable individuals can be matched by certain rule for the following crossover or mutation operation to produce a new generation population. If new generation not meet the user-defined requirement, a new iteration occurred for the next population. Once the new generation is convergence, the algorithm is ended and the final selected individuals are the best fitted. Figure 2 is the schematic illustration of general GA workflow.

\section{The Dislocation Recognition}

Traditional researchers have used the zero-point energy and electrostatic potential or used stress filter to detect the lattice

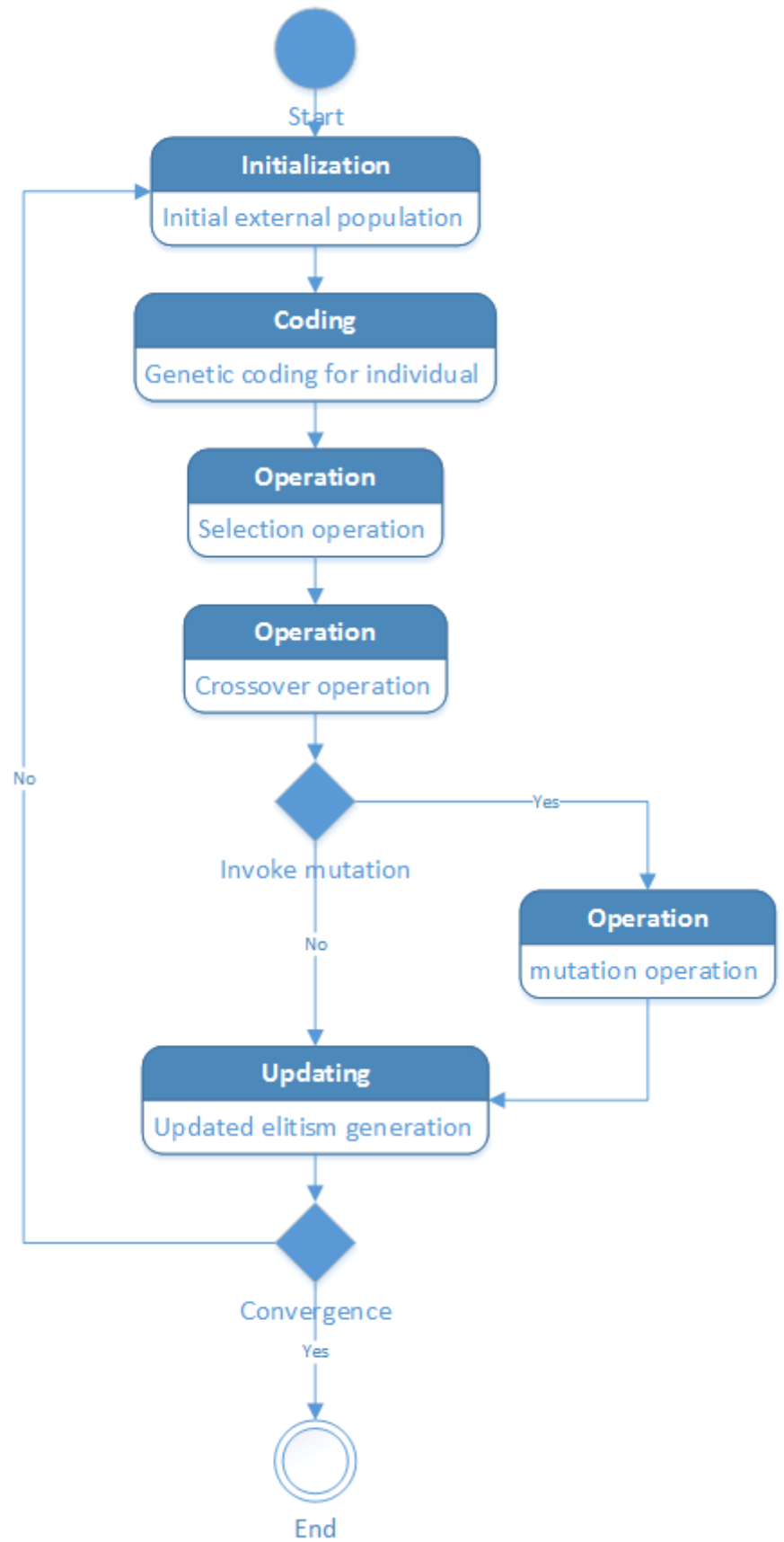

Fig. 2. Genetic Algorithm flowchart

deformation [6]. This traditional approach was proofed timeconsuming since the calculation was needed for each atom in the whole simulation box [7]. As the computer graphics technology development and accompany with the advantage of the computer core chips, new methods were developed. As reviewed in paper [8], there were various ways to identify the dislocation structure in the crystalline material except the tradition energy and stress filter approach. For example, the central symmetry parameter(CSP), neighbour distance analysis(NDA), common neighbour analysis(CNA). But among 
those methods, the most useful method for crystalline metal, in the author's opinion, is CNA since it works simply and directly. By comparing to the energy or stress filtering method, CNA can provide more detail information about the defect which can not be identified by energy contour plot. And also, it can generate better results compare against the CSP and NDA methods. Based on CNA, and associated with CSP, new methods like adaptive common neighbour analysis(A-CNA) and common neighbourhood parameter(CNP) are developed. The advantage of A-CNA against CNA is that it automatically derives the lattice parameter which is an input argues of CNA. For CNP, it proof to be more efficiency than CNA but more complicated.

\section{A. $C S P$}

The central symmetry parameter method is used to analysis the degree of distortion of lattice around an atom so that users can identify atoms in local structure whether they are in crystal defects such as dislocation or stack fault, perfect lattice or at the surface. The CSP is defined as:

$$
C_{c s p}=\sum_{i=1}^{N / 2}\left|R_{i}-R_{i+N / 2}\right|^{2}
$$

Where $\mathrm{N}$ is the number of those nearest atoms for centre atom, $R_{i}$ and $R_{i+N / 2}$ are vectors from the centre atom to the nearest atom $i$ and $i+N / 2$ separately. $N(N-1) / 2$ possible atom pair for the symmetrical crystal structure, but only the nearest $\mathrm{N}$ atoms included in this inspection parameter calculation. Given a FCC crystal structure, for example, the $\mathrm{N}$ is 12 for the number of nearest neighbours. Thus, total 66 possible atom pair will be included in the FCC central symmetric parameter definition. Respect to the result of CSP, it is proportional to the disorder rate of the lattice configuration. If the value is close to zero, this means the atom is in a perfect lattice. Otherwise, the greater CSP value means a greater defect in the current local system.

It's also notable that the disadvantage of CSP method is introduced by the intrinsic of this algorithm. The important one is that it can't be applied on the crystal structure which is not symmetric. Thus, the usage of CSP method is limited to few lattice structures. Another one is that it can't recognise the symmetrical deformation which caused by the increasing of lattice constant, such as thermal expansion. Except that, the result of CSP only reflect the deformation degree compare to the prefect lattice, but it can't give the information of defect type which is useful for the post analysis.

\section{B. NDA}

Neighbour distance analysis (NDA) [9] method is another dynamics analysis method which performs the structure defect recognition. A reference lattice configuration, which is important and directly influences the analysis result, introduced in this method. It considerate the reference configuration as the perfect lattice coordinates and compare the atom coordinates to the reference configuration to generate the analysis result $\lambda$, which is defined as:

$$
\lambda=\frac{1}{N} \sum_{i=1}^{N}\left|R_{i}-r_{i}\right|
$$

where $N$ is the number of nearest neighbour atoms of inspecting atom, $R_{i}$ is the distance vector from this atom to $i$ th neighbour atom in reference configuration. $r_{i}$ is the distance vector from this atom to $i$ th neighbour atom in current step frame.

The advantage of this NDA method, as shown in Equation 3 , is the flexibility of reference configuration. If take the first perfect frame as a reference frame, then the result of NDA method reflect the total deformation of the lattice structure. If consider the pre-step of the current frame as a reference frame each time, then the result will indicate the dynamics deformation during each simulation steps. But this would increase the computational cost and makes the analysis performance poor on the other side. Thus, it strongly recommends users only take this NDA analysis with fixed frame which is a perfect lattice configuration.

\section{CNA}

The CNA method was first introduced by Honeycutt [10] when he studied the melt and freeze of small cluster $\mathrm{Cu}$ by MD. The basic idea is to distinguish atoms structure by the common neighbours types and patterns. Later, Jonsson and Andersen [11], adopted the same method to study the glass crystalline structures produced by the phase transition from liquid to solid with Lennard-Jones fluids potential. And Schiøtz [12] researched the nanocrystalline copper by molecular dynamics simulation and examined the crystalline structure by CNA. He found that at elevated temperature, the CNA method was highly affected by the atom vibration by high temperature. If the system temperature is up to $300 \mathrm{~K}$, the cutoff distance is sensitive to the CNA result. For different Bravais lattice structure, the calculation of cutoff distance for CNA method is different. Usually, for close-packed structures, take FCC lattice as an example, their cutoff distance $r_{f c c}^{c u t o f f}$ is in the form:

$$
r_{f c c}^{c u t o f f}=\frac{1}{2}\left(\sqrt{\frac{1}{2}}+1\right) a_{f c c}
$$

For BCC structure and others, the cutoff distance is:

$$
r_{b c c}^{c u t o f f}=\frac{1}{2}(\sqrt{2}+1) a_{b c c}
$$

In the Honeycutt's approach, the neighbour atoms patterns(Fig. 4) in the bulk of crystal solid were identified with four indices a-b-c-d which decided by

a, the root pair atoms type( 1 or 2 for connected or disconnected)(Fig. 3),

b, common neighbour atom numbers of the root pair atoms,

c, bonds numbers in those common neighbour atoms,

$\mathrm{d}$, and another additional arbitrary value used to differentiate 


\begin{tabular}{|c||c|}
\hline jklm & Atom structure category \\
\hline 4211 & FCC \\
4220 & HCP \\
5522 & ICO \\
4422 & BCC4 \\
6622 & BCC6 \\
\hline
\end{tabular}

TABLE I

CNA INDICES PATTERN those have the same $\mathrm{a} b \mathrm{c}$ index number but different in bonding among common neighbours,

But in the implementation of this algorithm in the LAMMP$\mathrm{S}$, which is adopted from Faken [13] and Tsuzuki [14]. In the Faken's method, he treated the CNA method as a decomposition of radius distribution function(RDF) according to the surround of atom pairs between which the distance $r$ in the range of first peak of RDF $r_{c}$. And then three indices $\mathrm{jkl}$ will be assigned to these atoms pairs. The first index $\mathrm{j}$ and second index $\mathrm{k}$ are the same of the second and third index in Honeycutt's method. But the third index 1 is the number of bonds in the longest continuous chain formed by the $\mathrm{k}$ bonds between common neighbours. Thus' in Faken's method, it only calculates those pairs which are bonded. Comparing Faken's method with Voronoi analysis, it found that CNA method is easier to interpret the crystal structure since it quite insensitive to the small displacement of atom. Also, the Voronoi analysis results are singularities and sensitive to the atom displacement perturbations if the analysis system is the highly symmetric crystalline structure.

Tsuzuki [14] introduced a new approach which integrates the CSP and CNA into CNP. This method avoids the shortage of the CNA which is intricate to implement and to interpret and of the CSP which is only applicable in a centrosymmetric crystal. What's more, it inherits the advantage of CNA which is power for to analysis crystal structure and of CSP which is easy to implement and interpret. In this method, a parameter $Q_{i}$ (Equation 6) is defined for each atom $i$.

$$
Q_{i}=\frac{1}{n_{i}} \sum_{j=1}^{n_{i}}\left|\sum_{k=1}^{n_{i j}}\left(\mathbf{R}_{i k}+\mathbf{R}_{j k}\right)\right|
$$

Where $i, j, k$ are the atom index number, $\mathbf{R}$ is the vector connect the two atoms identified by the suffix from the first to second one.

While in the LAMMPS these indices jklm have slightly different usage.

$\mathrm{j}$, Number of common neighbours of atom $\mathrm{i}$ with each of its neighbours,

$\mathrm{k}$, Number of bonds between these common neighbours,

1, Maximum number of bonds of any common neighbours, $\mathrm{m}$, Minimum number of bonds of any common neighbours.

For each atom, the CNA indices jklm have the following sequence with different means and which shown in Table I.

In the LAMMPS CNA algorithm, for each atom, the nearest neighbour atoms list will be build first. And the CNA index

\begin{tabular}{|c||c||c|}
\hline number of neighbours & neighbour structure & atom structure \\
\hline 12 & 12 FCC & FCC \\
& 6 FCC 6 HCP & HCP \\
& others & ICO \\
\hline 14 & 4 BCC4 8 BCC6 & BCC \\
\hline \multicolumn{3}{c}{ TABLE II } \\
CNA STRUCTURE TABLE
\end{tabular}
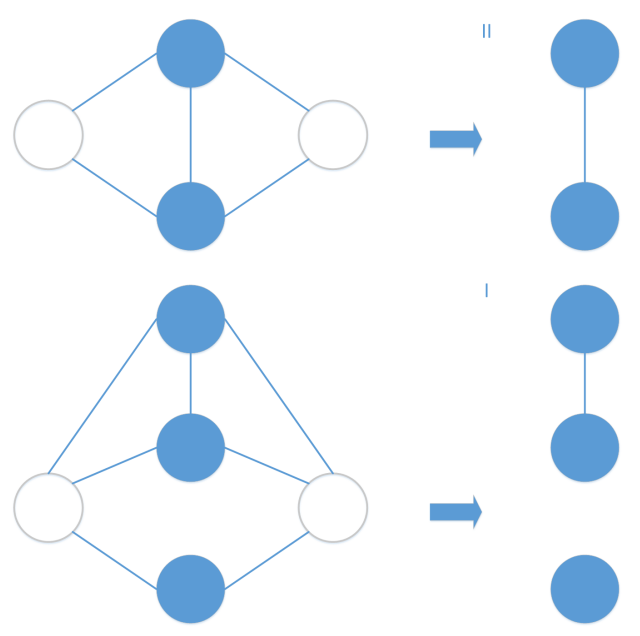

Fig. 3. Diagrammatic nomenclature for structural analysis technique. Nearestneighbours are connected by lines

will be allocated to each atom according to their displacement associated with its nearest neighbours. If the number of nearest neighbour is 12 , it means this atom is belonging a candidate of FCC, HCP or ICO structure lattice category. Else if the number of nearest atoms is 14, it means this atom is in the BCC candidate list. For each candidate in FCC and HCP list, the nearest neighbours pattern of CNA indices will be matched via Table I. And finally, the atom's structure is determined by the common neighbour atoms' CNA indices sequence via Table II.

\section{A-CNA}

The adaptive-common neighbour analysis method is based on CNA method but the different is the flexibility of cutoff distance which can be detected by the A-CNA for each particle while it is fixed in CNA method [9]. Since the cutoff distance is calculated automatically, it can inspect those atoms on the surface which can't be analysis by CNA method.

The algorithm of A-CNA is a recursive method which considers a general maximum number of atom neighbours for all possible structure. Another issue is it match the standard Bravais structures one by one for each atom by use the experiment equation of cutoff distance. For example, the local cutoff distance for FCC structure is defined in Equation 7.

$$
r_{\text {cut }}^{\text {local }}=\frac{1+\sqrt{2}}{2} \cdot \frac{\sum_{j=1}^{12}\left|r_{j}\right|}{12}
$$

Where the $r_{j}$ is the distance of atom $j$ to the central atom. It will consider 14 and 12 nearest neighbour atoms for BCC and $\mathrm{FCC}(\mathrm{HCP})$ structure, separately. The computational cost 


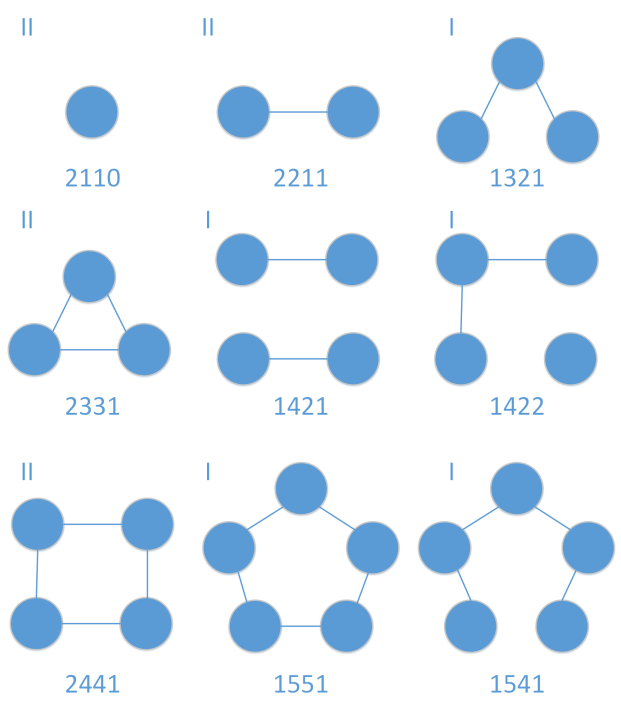

Fig. 4. common diagram in dense atom system

of A-CNA is definitely higher compare against the result of CNA method according to the recursive algorithm of local cutoff distance determination. From the practical test, it found the performance is only $25 \%$ expensive than standard CNA method [9] when identifying BCC, FCC, HCP structure.

\section{THE COMPUTATIONAL INTELLIGENCE DISLOCATION RECOGNITION METHOD}

A computational intelligence dislocation recognition method is present in this section. This approach is based on the CNA method with the computational intelligence algorithm and suite for FCC lattice structure material. The general idea is to calculate the CNA parameter for each atom, and then distribute the different CNA parameter to each atom and then use the GA based algorithm to generate the dislocation. As known, the edge dislocation is consist of dislocation line, dislocation plan, and dislocation slip direction. The dislocation line is a list of atoms which is the edge of the half atom plane like a knife cut in the perfect crystal structure. In CNA method, if an atom has 12 nearest neighbours, then this atom is in one of the following lattice structure, FCC, Hexagonal close-packed(HCP) and icosahedral structure. If there are 6 atoms have the following structure in $i j k l-4211$ which indicates an FCC structure, and others 6 atoms have 4220 which indicates an HCP structure, then, this atom maybe is in a dislocation core.

The difficult to identify the dislocation is not in the atom structure classification. The problem is to determine the dislocation plane and dislocation edge by the scattered atom information. The dislocation atom must be connected. And what's more, the dynamics of the dislocation edge which is caused by the external stress or strain on the system. This makes the recognition more complicated than the static situation.
Assume all of the atoms in the simulation box are in the perfect Bravais lattice position. The simulation box is under external loads such as stress or strain. After a few steps simulation, the $i$ th atom who has $M$ nearest neighbours is been examined and the vector of this atom to the $j$ th neighbour atom $r_{i j}$ changes according to the external loads. By applying the CNA method reviewed in Section 3-3, total $K$ distortion atoms can be identified. Denote the distortion atoms as set $\mathbf{Z}$ in which distorted atoms defined as $a_{i}$. The problem is to find the subset of $\mathbf{Z}$ in which each individual's vector is out of the sequence of perfect lattice configuration and the distance between them is minimum. Now coding the chromosome of each individual in the population of the first generation as the bounding box of each distorted atoms which detected by the CNA method. The bounding box can be depicted as:

$$
B=\left\{X_{\min }, Y_{\min }, Z_{\min }, X_{\max }, Y_{\max }, Z_{\max }\right\}
$$

$$
B_{s}=\{X-r, Y-r, Z-r, X+r, Y+r, Z+r\}
$$

where $X_{\min }, Y_{\min }, Z_{\min }, X_{\max }, Y_{\max }, Z_{\max }$ are the minimum and maximum of bounding box's coordinates. For a single atom, the bounding box is defined as in Equation 9, where $X Y Z$ are the atom's coordinate and $r$ is the radius of a single atom.

With the GAs methodology as described in Section 2 and for the application in this problem, define the genetic as the coordinate of the bounding box. After that, in this scenario, the algorithm is selecting the superiors individual to survive and then crossover to generate a new generation of individuals till the population is convergence to limited ones that can't meet the requirement of the fitness function. Thus, this function $f$ for each individual can be defined as following:

$$
f_{i}=\frac{1}{\min \left(R_{i j}\right)}<R_{c}
$$

$$
\begin{aligned}
& \text { MAX: } f_{i}=\sum_{i=1}^{N} \sum_{j=i+1}^{N} \min \left(R_{i j}\right) \\
& =\sum_{i=1}^{N} \sum_{j=i+1}^{N} \min \left(\sqrt{\left(x_{i}-x_{j}\right)^{2}+\left(y_{i}-y_{j}\right)^{2}+\left(z_{i}-z_{j}\right)^{2}}\right)
\end{aligned}
$$

Where $R_{i j}$ is the distance between each individual. At first, calculate the total fitness of all individual, and get the relative fitness of each individual for the probability for next generation. $R_{c}$ is the predefined cutoff distance between two individual. The fitness calculation workflow is illustrated in Figure 5.

The total fitness $F$ is:

$$
F=\sum_{i=1}^{K} f_{i}=\sum_{i=1}^{K} \frac{1}{\min \left(R_{i j}\right)}
$$




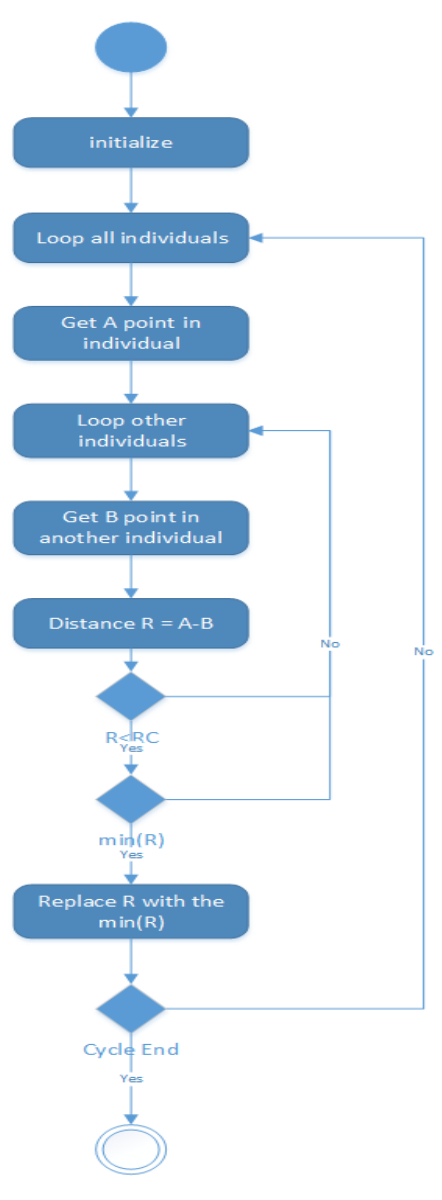

Fig. 5. Fitness calculation work flow

After the calculation of individual fitness and total fitness, the probability $P$ can be defined as the fraction between the two values.

$$
P=\frac{f_{i}}{F}=\frac{1}{\min \left(R_{i j}\right) \sum_{i=1}^{K} \frac{1}{\min \left(R_{i j}\right)}}
$$

After selection of those superiors for next generation, the crossover operation applied on them who are meet the crossover requirement and another generation of new individuals was produced. This mate operator is defined as an union operation between two individuals whose distance is smaller than the minimums neighbour distance as illustrated in Figure 6. After this crossover operation, the new generation's genetic is changed according to the fitness function requirements and the dislocation cores are growing with the new generation. After a few step's iteration, the dislocation can be identified till the new iteration is convergence.

\section{RESUlT}

By implemented this algorithm in LAMMPS simulation, the result seems more efficient than traditional recognition method. The test which is an example in LAMMPS software and running with a model of 4056 Nickel atoms has been shown in Figure 7. The boundary condition is set as periodical

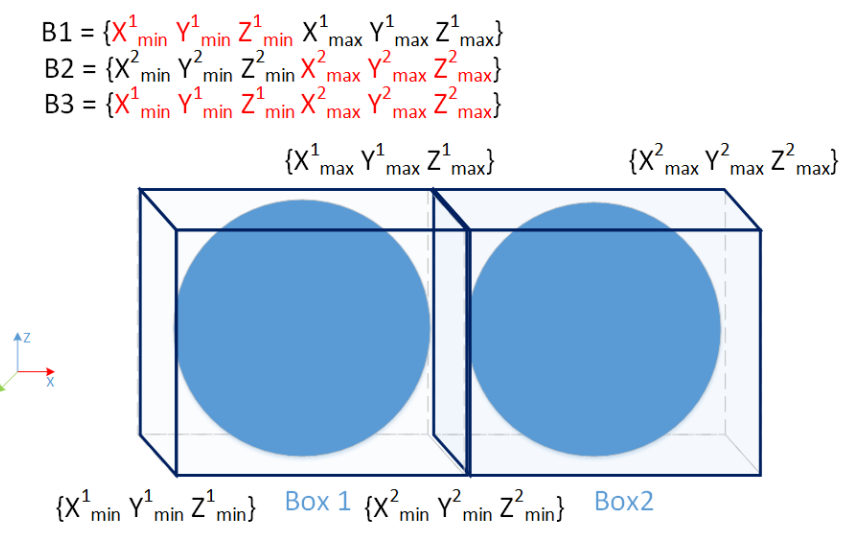

Fig. 6. An example illustration of crossover operation, in which a new generation individula $\mathrm{B} 3$ is produced by selecting the minimums $\mathrm{X}$ Y Z coordinates from individual $\mathrm{B} 1$ and the maximum $\mathrm{X}$ Y $\mathrm{Z}$ coordinates from individual B2.

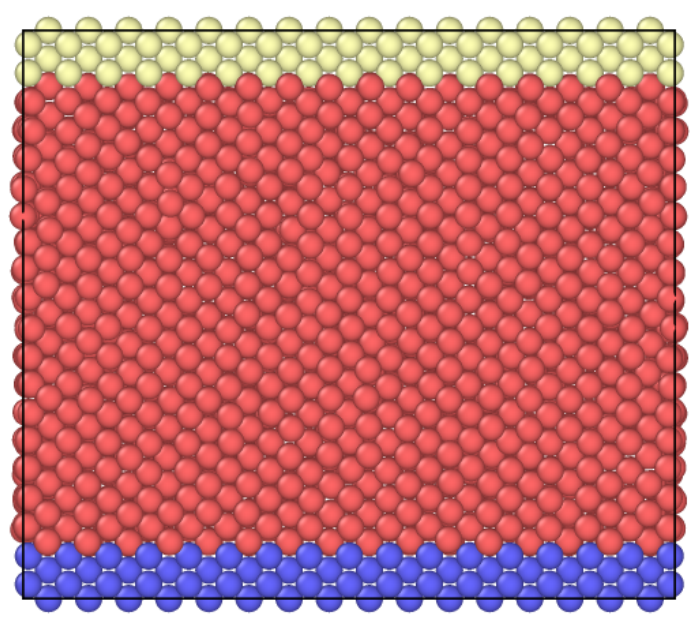

Fig. 7. The simulation configuration

condition in $\mathrm{X}$ and $\mathrm{Z}$ directions, but as shrink condition in $\mathrm{Y}$ direction. And the system is under shear deformation in $\mathrm{X}$ direction by set the ramp velocity along the $\mathrm{Y}$ direction. The simulation step is set to 0.001 ps for a 3000 steps loops for 4 times. During the simulation, after 1200 steps, the initiation of dislocations are detected till the simulation end. The result in the middle steps is provided and is shown in the Figure 8. In step 2000, the first generation of 486 atoms is recognised as distorted atoms of candidate dislocation. After 7 iterations, total 20 dislocations in which one individual with a maximum of 90 atoms is recognized. After the first crossover, the population decreased sharply because of the cutoff distance have been set for the minimum neighbour distance between two atoms. By investigating other steps (step 2100 and step 2200) recognition, it is found that the maximum iteration does not exceed 8 times before all of the dislocations have been recognised.

By comparing with the CNA result as shown in Figure 9, the 


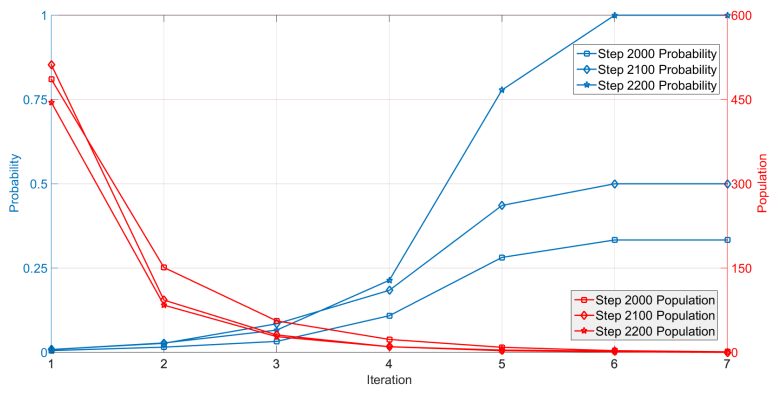

Fig. 8. The plot of iteration v.s. probability and population

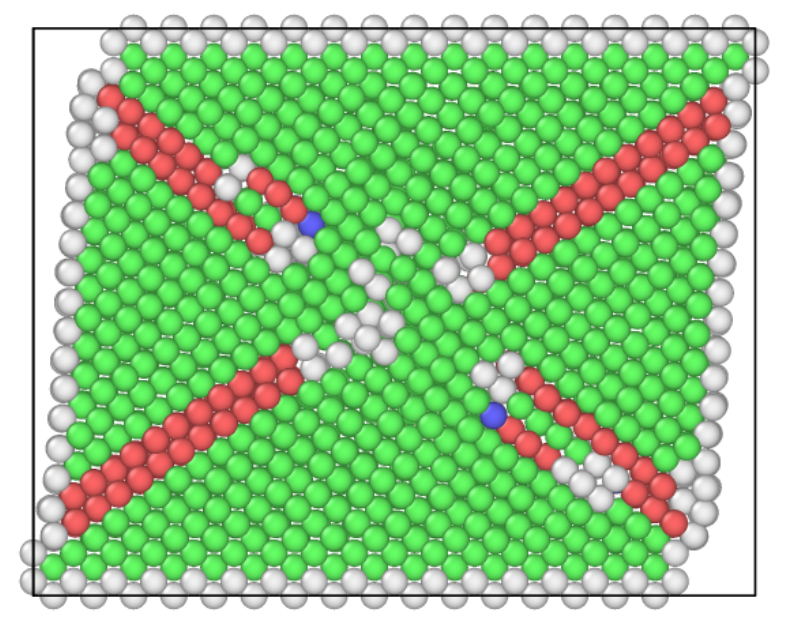

Fig. 9. CNA result

result(Figure 10) is more accurate and clear to distinguish each dislocation by different colours, Also, the dislocation plane and direction can be calculated and recognised associated with those information.

\section{CONCLUSION AND FUtURE WORK}

In this paper, a genetic algorithm inspired dislocation recognition method is presented with the acceptable result which agrees with the CNA method. This algorithm demonstrated in this paper is a GA inspired method since it not fully following the GA framework.Thus, in the future, the GA inspired dislocation recognition should be revised and evolved by improve the fitness function, select operation, etc. to make the result more accurate and improve the performance of this algorithm. Also, it is not been tested in the full range with more complicated cases. It's our responsibility to validated this algorithm through a completely way in the future.

The dislocation recognition is only the beginning step of the journal of material science in the microscope modelling and simulation. The dislocation motivation and interaction is essential for understanding the influence of dislocation on material mechanical properties. More important, our future work is to investigate the dynamic of dislocation in the atomic level.

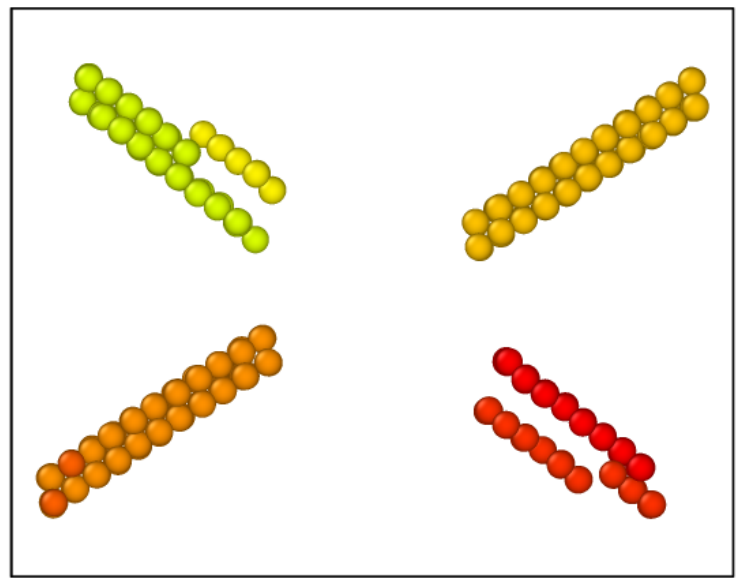

Fig. 10. Dislocation recognition result, different colour presents different dislocation

\section{ACKNOWLEDGMENT}

The authors would like to acknowledge the scholarship award to the first author provided by the SCHOOL OF ENGINEERING AND BUILT ENVIRONMENT, GLASGOW CALENDONIAN UNIVERSITY. And this work was also supported by National Natural Science Foundation of China (Grant No. 51405044). The authors thank COLLEGE OF MECHANICAL ENGINEERING, CHONGQING UNIVERSITY.

\section{REFERENCES}

[1] G. I. Taylor, The Mechanism of Plastic Deformation of Crystals. Part I. Theoretical, Proceedings of the Royal Society of London, Series A 145 (855): 362-387, 1934.

[2] Holland JH., Adaptation in natural and artificial systems, The University of Michigan Press; 1975.

[3] De Jong KA., An analysis of the behavior of a class of genetic adaptive systems, Doctoral dissertation, University of Michigan; 1975.

[4] Yi Chen, Yong Mab, Zheng Lu, Lixia Qiu, Jin He,Terahertz spectroscopic uncertainty analysis for explosive mixture components determination using multi-objective micro-genetic algorithm, Advances in Engineering Software, 42, 649-659, 2011.

[5] Yi Chen, Bei Peng, Multi-objective optimization on multi-layer configuration of cathode electrode for polymer electrolyte fuel cells via computational-intelligence-aided design and engineering framework, Applied Soft Computing, 43, 357-371, 2016.

[6] Sameep Mehta, Raghu Machiraju, et al. Detection and Visualization of Anomalous Structures in Molecular Dynamics Simulation Data,To be done-check the journal name, 2004.

[7] R.J. Needs, W.K. Leung and G. Rajagopal, Calculation of silicon self interstitial defects, Physical Review Letters vol. 83, 1999.

[8] Li D, Wang F C, Yang Z Y, et al. How to identify dislocations in molecular dynamics simulations?, Sci China-Phys Mech Astron, 57: 2177-2187, doi: 10.1007/s11433-014-5617-8, 2014.

[9] H. Alexander Stukowski, Structure identification methods for atomistic simulations of crystalline materials, Modelling and Simulation in Materials Science and Engineering, vol. 20, 2012.

[10] J. Dana Honeycutt, Hans C. Andemen, Molecular Dynamics Study of Melting and Freezing of Small Lennard- Jones Clusters, J. Phys. Chem, Vol. 91, 4950-4963, 1987.

[11] H. Jonsson, H.C. Andersen, Icosahedral ordering in the Lennard-Jones liq-uid and glass, Phys. Rev. Lett. 60, 2295-2298, 1988.

[12] J. Schiøtz, T. Vegge, F. D. Di Tolla and K. W. Jacobsen, Atomic-scale simulations of nanocrystalline metals, To be done-check the journal name, 1999. 
[13] Faken, D. and Jonsson, H. Systematic analysis of local atomic structure combined with $3 D$ computer graphics, Computational Materials Science, Vol 2, 279-28, 1994.

[14] H. Tsuzuki, P. S. Branicio, J. P. Rino, Structural characterization of deformed crystals by analysis of common atomic neighborhood, Computer Physics Communications, 177(6): 518- 523, 2007. 